\section{Interfering with interferon}

\section{By Lauren Martz, Staff Writer}

Type I interferon receptors are key factors that promote antiviral immunity, but two separate teams have found that elevated interferon signaling may actually be detrimental to the body's ability to fight persistent infections. Groups at The Scripps Research Institute and the University of California, Los Angeles showed that blocking type I interferon during the persistent stages of viral infections in mice can resolve the infections. ${ }^{1,2}$

One key implication from the findings is that researchers should re-evaluate how to use interferon in treating $\mathrm{HCV}$, as it has important antiviral benefits in acute infections but may be damaging during persistent infection.

According to David Brooks, who led the UCLA team, "There is a growing idea that dampening the constant immune activation in chronic infectious diseases has benefits. Our idea that interferon may be driving persistent infections adds one more piece to the puzzle." Brooks is assistant professor of microbiology, immunology and molecular genetics at UCLA.

Type I interferon is secreted by multiple cell types, including immune cells, and plays a key role in the antiviral immune response. Many acute viral infections are cleared by the antiviral cytotoxic $T$ cell response that involves interferon signaling.

But some viruses, including HIV and $\mathrm{HCV}$, establish persistent infections by wearing down the immune system via chronic inflammation and $\mathrm{T}$ cell exhaustion or by evading the immune system through the upregulation of immunosuppressive factors that dampen antiviral immunity.

Indeed, previous studies have shown that upregulation of immunosuppressive factors such as programmed cell death 1 ligand 1 (CD274 molecule; PD-L1; B7-H1) or $I L-10$ contribute to chronic infections and cancers, ${ }^{3}$ and other work suggests that chronic inflammation contributes to chronic infection. ${ }^{4}$

Now, two separate teams have built on the prior findings by examining immune system dysfunction in chronic diseases. Both teams concluded that inhibiting interferon receptor signaling, despite known antiviral functions, may actually help clear viruses in the persistent stage of infection.
"There is a growing idea that dampening the constant immune activation in chronic infectious diseases has benefits. Our idea that interferon may be driving persistent infections adds one more piece to the puzzle."

-David Brooks, University of California, Los Angeles

A group from Scripps led by Michael Oldstone found that antibodymediated inhibition of type I interferon reduced chronic inflammation, immune suppression and viral load in mice with persistent viral infections.

Oldstone is professor of immunology and microbial science at Scripps. The team also included researchers from the Washington University in St. Louis School of Medicine.

The group infected mice with two lymphocytic choriomeningitis virus (LCMV) strains_Cl13 and Armstrong-to model human viral infections. $\mathrm{Cl} 13$ lasted more than 90 days and thus modeled persistent infections, whereas the Armstrong strain served as a control because it caused an acute infection that was cleared within 8 days.

In the Cl13-infected mice, serum interferon levels, dendritic cell production of interferon- $\beta$ (IFNB; IFN- $\beta$ ) and proinflammatory cytokine levels were higher than those in mice infected with the Armstrong strain.

Cl13 mice receiving a prophylactic anti-type I interferon receptor antibody had lower levels of proinflammatory cytokines and chemokines and negative immune regulatory elements PD-L1 and IL-10 than untreated mice. These findings suggested that impeding interferon ignaling could reduce features of immune system dysfunction ponsible for persistent infection.

flactic treatment with anti-interferon receptor antibodies led animals and caused an overall increase in viral burden in the control mice.

These findings confirmed that type I interferon does have its usesparticularly during acute infection-but also contributes to immune dysfunction later in cases of persistent infections.

The anti-interferon receptor antibodies also restored normal lymphoid architecture and were effective at clearing the virus after the onset of persistent infection.

Finally, the team found that the antibodymediated clearance of Cl13 LCMV required $\mathrm{CD}^{+} \mathrm{T}$ cells. In the treated mice, depletion of CD4 prevented the accelerated viral clearance by anti-interferon antibodies.

\title{
Replication
}

Separately, a group led by Brooks obtained similar results and drew the same conclusions about the dual roles of interferon in viral infections.

Brooks is a former postdoctoral fellow in Oldstone's lab. The group also included researchers from the Cincinnati Children's Research Foundation and the University of

\section{Cincinnati College of Medicine.}

The UCLA group used the same mouse models and found that type I interferon-stimulated genes and regulatory factors were initially expressed at the same levels in the acute and chronic infection models, but their levels were elevated during persistent infection. 
The team also found that blocking the interferon receptor decreased chronic inflammatory signaling and immunosuppressive components during persistent infection. Cl13-infected mice receiving an antiinterferon receptor antibody had viral titers that by day 30 were either lower than those in mice given an isotype control antibody or cleared.

Similar to the Scripps findings, delivery of the antibody prior to persistent $\mathrm{Cl} 13$ infection of mice led to virus clearance during persistent infection, whereas delivery of the antibody during acute Armstrong infection of mice led to virus persistence.

The team also detected elevated levels of $\mathrm{CD} 4^{+} \mathrm{T}$ cells and type II interferon- $\gamma$ (IFNG; IFN- $\gamma$ ) during persistent blockade of either CD4 or IFN- $\gamma$ prevented the accelerated viral clearance by the anti-type I interferon receptor antibody.

Also, treatment with anti-interferon receptor antibody starting 25 days after infection accelerated viral clearance, suggesting that type I interferon blockade could be used to treat established persistent infections.

Both studies were published in Science. infections and found that antibody-mediated

"HCV is particularly interesting because a subset of patients is actually given interferon as a drug. In those patients, when interferon treatment fails, it is usually because they already had a high enough level of interferon to cause immune dysfunction. In these cases, adding interferon is like bringing sand to the beach. It doesn't help, and our findings suggest it may actually hurt them," he said.

Nicolette said that the problem with anti-interferon for HCV is that it is already possible to eradicate the infection with type I interferon in some patients. "It drives the immune system to higher activity," he said.

"Thinking about developing these kinds of interferon-targeted therapies is really a challenge because it is counterintuitive. You want to disable an antiviral mechanism in patients with a virus, but this type of treatment may induce an immunological change that tips the scales in favor of virus clearance," said Nicolette.

He added that it would probably be very hard to find an infectious disease doctor willing to treat patients with a strategy that is in direct opposition to a proven approach.

Regardless of indication, Brooks said, "a lot more studies are needed. Interferon is so important in regulating the immune response

\section{Chronicles of interferon}

Subpopulations of patients with HIV or HCV may be the most likely to benefit from anti-interferon therapies.

According to Brooks, "The next step is to validate the data in infections such as HIV."

Charles Nicolette, CSO and VP of R\&D at Argos Therapeutics Inc., told SciBX that "HIV may be a good choice to move forward in development. Specifically, patients with high levels of chronic inflammation driven by type I interferon would be key populations."

Argos' AGS-009, an anti-IFN- $\alpha$ (IFNA) antibody, recently completed Phase I testing for systemic lupus erythematosus (SLE).

Rafick-Pierre Sékaly, co-director and scientific director of the Vaccine \& Gene Therapy Institute of Florida, added that patients with HIV who are nonresponsive to antiretroviral therapy generally show high interferon signatures and high levels of PD-L1.

"HIV patients not on an antiretroviral therapy, and even those patients on therapy who may be experiencing an ongoing smoldering immune activation that chronically leads to immune senescence, may benefit from inhibition of the negative effects of interferon signaling," said Brooks.

Nicolette said other benefits of anti-interferon therapy include "providing relief from the damaging effects of chronic inflammation and reprograming the immune system to better respond to the infection."

In addition to HIV, the authors of the Science papers think some patients with HCV could benefit from anti-interferon therapy, although it may be more difficult in the HCV setting to translate the strategy into clinical trials.

Brooks said his team does not have ongoing HCV studies but thinks the findings could be applicable to the disease. to infection as well as regulating homeostasis. One really has to tease apart these functions in interferon signaling to identify the best way to target it clinically. We need to preserve the antiviral role of interferon but not the immune dysfunction and chronic activation."

Brooks said UCLA has filed for a patent covering his team's work. The IP is available for licensing. The patent and licensing status for the findings from the Scripps group is unavailable.

Martz, L. SciBX 6(16); doi:10.1038/scibx.2013.381

Published online April 25, 2013

\section{REFERENCES}

1. Teijaro, J.R. et al. Science; published online April 12, 2013; doi:10.1126/science.1235214

Contact: Michael B.A. Oldstone, The Scripps Research Institute, La Jolla, Calif.

e-mail: mbaobo@scripps.edu

2. Wilson, E.B. et al. Science; published online April 12, 2013; doi:10.1126/science.1235208

Contact: David G. Brooks, University of California, Los Angeles, Calif.

e-mail:dbrooks@microbio.ucla.edu

3. Appay, V. \& Sauce, D. J. Pathol. 214, 231-241 (2008)

4. Zinselmeyer, B.H. et al. J. Exp. Med. 210, 757-774 (2013)

COMPANIES AND INSTITUTIONS MENTIONED

Argos Therapeutics Inc., Durham, N.C.

Cincinnati Children's Research Foundation, Cincinnati, Ohio

The Scripps Research Institute, La Jolla, Calif.

University of California, Los Angeles, Calif.

University of Cincinnati College of Medicine, Cincinnati, Ohio

Vaccine \& Gene Therapy Institute of Florida, Port St. Lucie, Fla.

Washington University in St. Louis School of Medicine, St. Louis, Mo. 